\section{Reply to Is progesterone a neutral or protective factor for breast cancer?}

\author{
Cathrin Brisken
}

I am grateful for the comments on my Review article (Progesterone signalling in breast cancer: a neglected hormone coming into the limelight. Nature Rev. Cancer 13, 385-396 $(2013))^{1}$ by Paola Muti (Is progesterone a neutral or protective factor for breast cancer? Nature Rev. Cancer http://dx.doi.org/10.1038/ nrc3518-c1 (2014)) $)^{2}$, as they provide me with the opportunity to clarify a few points and to reiterate the fundamental biological principle that signalling outcome depends on whether the stimulus is transient or sustained and also the context in which it occurs: for instance, in a premenopausal or postmenopausal woman.

Muti suggests that the Review supports the hypothesis that increased levels of endogenous serum progesterone might represent a risk factor for breast cancer. This is not the case. I did not comment about absolute serum progesterone levels but linked the time of exposure to progesterone and the resulting recurrent activation of progesterone receptor signalling during a woman's life to increased breast cancer risk. More specifically, I referred to the recurrent but transient increases in serum progesterone levels related to menstrual cycles for premenopausal women and the administration of oestrogens and progestins (that is, synthetic progesterone receptor agonists) in the context of hormone replacement therapy (HRT) for postmenopausal women.

As Muti explains, determining serum hormone levels is complicated and can be confounded by a multitude of factors. In this context, I found the recent study by Muti and colleagues $^{3}$ very interesting, where the large ORDET (Hormones and Diet in the Etiology of Breast Cancer) cohort was successfully used to show that serum levels of free testosterone are associated with breast cancer risk in premenopausal women but not progesterone or any other endogenous steroid.

Muti also suggests that I did not include evidence for progesterone as a protective factor. The in vivo study by Foidart et al. ${ }^{4}$ quoted in this context examines a very limited number of patients. Forty postmenopausal women received topical treatment with a gel containing placebo $(n=10)$, oestradiol $(n=10)$, progesterone $(n=13)$ or oestradiol and progesterone $(n=7)$ for 14 days before breast surgery for aesthetic reasons or for a benign lesion. The authors report that oestradiol induced cell proliferation, which was reduced by concomitant administration of progesterone. How the observed cell proliferation in the postmenopausal breasts in this 2-week experiment with topical application relates to breast cancer risk that results from years of HRT is unclear. It is well established that the relative risk related to HRT with oestradiol only is negligible and so is the relative risk related to combined oestradiol and natural progesterone administration. The combination of oestradiol and synthetic progestins, which does increase breast cancer risk, was not tested. (It is unclear whether the negative effects of the synthetic compounds versus natural progesterone relate to their longer half-lives and the resulting ability to elicit more sustained signalling than natural occurring progesterone and/or to the fact that the synthetic compounds are not completely selective and can also interact with other nuclear hormone receptors, such as the androgen or the glucocorticoid receptor.)

Muti further quotes in vitro studies with the hormone receptor-positive breast cancer cell line MCF-7 (REFS 5,6) and a hormone receptor-negative breast cancer cell line, MDA-MB-231, transfected with the progesterone receptor ${ }^{7}$, in which progesterone inhibits cell proliferation ${ }^{5-7}$. The extrapolation of in vitro findings from cancer-derived cell lines under specific experimental conditions to the complex and dynamic in vivo situation is problematic; hence I only referred to in vivo data in the Review. Moreover, in these in vitro models, typically close to $100 \%$ of the cells express the progesterone receptor, whereas in vivo the majority of mammary epithelial cells do not. As discussed in the Review, most of the cell proliferation that is triggered by progesterone occurs by paracrine mechanisms in the progesterone receptor-negative cell compartment.

Although this point was not raised by Muti, I should add that I glossed over the important epidemiological observation that early full-term pregnancies have a protective effect with regards to breast cancer risk; this omission was made owing to space limitations. Women who have their first baby before age 20 have $50 \%$ of the risk of nulliparous women and $40 \%$ of that of women whose first birth is after age 35 (REF. 8). The mechanisms underlying this protection are an area of intense investigation with a special emphasis on stem cells, and this discussion was beyond the scope of the Review.

During pregnancy, many hormones are increased for a sustained period. Serum progesterone levels are particularly high; in the third trimester they can reach $180 \mathrm{ng} \mathrm{ml}^{-1}$, as opposed to the $8-33 \mathrm{ng} \mathrm{ml}^{-1}$ in luteal phase and $0.1-0.8 \mathrm{ng} \mathrm{m}^{-1}$ in follicular phase. Indeed, this very high and sustained exposure to progesterone ultimately contributes to protection. The protective effect of pregnancy is also observed in mice and rats. Interestingly, it can be mimicked by administration of $17-\beta$-oestradiol and progesterone at pregnancy levels over 21 days. Thus, in conjunction with oestradiol levels that are 30-40 times higher than peak levels in the menstrual cycle, sustained exposure to pregnancy levels of progesterone provides protection early in life. These findings highlight that hormone action is highly context-dependent and that the biological effects of progesterone depend on the dose, the duration of the stimulation (9 months of pregnancy versus 2 weeks of luteal phase during the menstrual cycle), the specific kinetics, the presence of concomitant high levels of oestradiol and the woman's age.

Cathrin Brisken is at ISREC - Swiss Institute for
Experimental Cancer Research, School of
Life Sciences, Ecole Polytechnique Fédérale
de Lausanne (EPFL), SV2.832 Station 19,
CH-1015 Lausanne, Switzerland.
e-mail: cathrin.brisken@epfl.ch doi: 10.1038/nrc3518-c2

1. Brisken, C. Progesterone signalling in breast cancer: a neglected hormone coming into the limelight. Nature Rev. Cancer 13, 385-396 (2013).

2. Muti, P. Is progesterone a neutral or protective factor for breast cancer? Nature Rev. Cancer http://dx.doi.org/10.1038/nrc3518-c1 (2014).

3. Schernhammer, E. S. et al. Endogenous sex steroids in premenopausal women and risk of breast cancer: the ORDET cohort. Breast Cancer Res. 15, R46 (2013).

4. Foidart, J. M. et al. Estradiol and progesterone regulate the proliferation of human breast epithelial cells. Fertil. Steril. 69, 963-969 (1998).

5. Alkhalaf, M., El-Mowafy, A. \& Karam, S. Growth inhibition of MCF-7 human breast cancer cells by progesterone is associated with cell differentiation and phosphorylation of Akt protein. Eur. J. Cancer Prev. 11, 481-488 (2002)

6. Alkhalaf, M. \& El-Mowafy, A. M. Overexpression of wild-type $\mathrm{p} 53$ gene renders MCF-7 breast cancer cells more sensitive to the antiproliferative effect of progesterone. J. Endocrinol. 179, 55-62 (2003).

7. Lin, V. C., Eng, A. S., Hen, N. E., Ng, E. H. \& Chowdhury, S. H. Effect of progesterone on the invasive properties and tumor growth of progesterone receptortransfected breast cancer cells MDA-MB-231. Clin. Cancer Res. 7, 2880-2886 (2001).

8. MacMahon, B. et al. Age at first birth and breast cancer risk. Bull. World Health Organ. 43, 209-221 (1970).

Competing interests statement

The author declares no competing interests. 